\title{
Structure of Personality at Intrapsychic and Interpersonal Levels and Depression of Caregivers of Patients Affected by Alzheimer's Disease: Which Psychotherapeutic Approach?
}

\author{
Giulietti Maria Velia ${ }^{1}$, Anna Vespa ${ }^{2 *}$, Stefano Berti ${ }^{3}$, Guido Gori ${ }^{4}$, Marica Ottaviani ${ }^{2}$, \\ Cristina Meloni' ${ }^{1}$, Paolo Fabbietti ${ }^{5}$, Giuseppe Pelliccioni ${ }^{1}$, Lucia Paciaroni ${ }^{1}$, Roberta Spatuzzi ${ }^{6}$ \\ ${ }^{1}$ Department of Research, INRCA-IRCCS National Institute of Science and Health for Aging, Ancona, Italy \\ ${ }^{2}$ Department of Research, Scientific and Technological Area, INRCA-IRCCS National Institute of Science and Health for Aging, \\ Ancona, Italy \\ ${ }^{3}$ Health Promotion Department, Ancona \\ ${ }^{4}$ Alzheimer Daily Center, Florence, Italy \\ ${ }^{5}$ Biostatistical Center, INRCA-IRCCS National Institute of Health and Science on Aging, Ancona, Italy \\ ${ }^{6}$ U.O.C. Hospice/Palliative Care Departments, Potenza, Italy \\ Email: giulietti.mariavelia@libero.it, ^a.vespa@inrca.it,marica_O@libero.it, stefano.berti@sanita.marche.it, \\ melcri86@hotmail.it,p.fabbietti@inrca.it,g.pelliccioni@inrca.it,roberta.spatuzzi@yahoo.com
}

How to cite this paper: Velia, G.M., Vespa, A., Berti, S., Gori, G., Ottaviani, M., Meloni, C., Fabbietti, P., Pelliccioni, G., Paciaroni, L. and Spatuzzi, R. (2018) Structure of Personality at Intrapsychic and Interpersonal Levels and Depression of Caregivers of Patients Affected by Alzheimer's Disease: Which Psychotherapeutic Approach? Advances in Alzheimer's Disease, 7, 103-118.

https://doi.org/10.4236/aad.2018.74008

Received: September 7, 2018

Accepted: November 19, 2018

Published: November 22, 2018

\begin{abstract}
Background: Many studies have underlined as caregiving for people with Alzheimer's disease (AD) is highly stressful and has significant negative consequences. Objectives: The study of the structure of personality, can help to understand the association between depression, intrapsychic and interpersonal processes of caregivers of Alzheimer's disease patients and what kind of intervention can be planned to favor the stress burden management. Methods: Case group: Caregivers (CG) $(\mathrm{n}=75)$; control group, Subjects not Caregivers (nCG) (n. 104). Tests: SASB questionnaire (Structural Analysis of Interpersonal Behavior) describing intrapsychic and interpersonal processes of the structure of personality validated on the basis of DSMIV; CDQ questionnaires-depression. Results: Intrapsychic level: From the results it emerged that CGs had lower autonomy in their choices, and lower acceptance of their own feelings, and exercised greater self-control exhausting themselves toward predetermined goals, and more depression compared to the control group. They may be not able to achieve psychic equilibrium in the presence of stress: they may likely become disoriented and engage in behaviors that may be self-defeating. SASB-Cl = Autonomy $(\mathrm{p}<0.001)$; SASB-Cl2-Autonomy and Love $(\mathrm{p}<0.001)$, SASB-CL3-Love $(\mathrm{p}<0.001)$; SASB-Cl4 Love and con-
\end{abstract}


Copyright (c) 2018 by authors and Scientific Research Publishing Inc. This work is licensed under the Creative Commons Attribution International License (CC BY 4.0). http://creativecommons.org/licenses/by/4.0/ trol ( $\mathrm{p}<0.001)$, SASB-CL5-Control $(\mathrm{p}=0.015)$, SASB-Cl6-Control and hate $(\mathrm{p}<0.001)$, SASB-Cl7-Hate $(\mathrm{p}<0.001)$. Conclusions: Intrapsychic characteristics such as tendency to depression, inability to being in contact with their own feelings, excessive self-control may be linked to difficulties in facing burden of care and indicate serious difficulties to adaptation to burden condition. The knowledge of these modalities could allow to plan a psychotherapeutic and multidisciplinary intervention aimed at facing and overcoming the psychological distress of the caregiver.

\section{Keywords}

Caregiver, Alzheimer, Depression, Intrapsychic Behaviours, Interpersonal Behaviours, Personality

\section{Introduction}

To assist a person with dementia it can wear out emotional resources and the caregiver can show anxious-depressive symptoms [1] with somatic troubles that often limit relief abilities [2] [3] [4] [5] [6]. A progressive illness such as the Alzheimer's disease leads to a deep change in the life style of the whole family system. The changes imposed by the progression of illness pose different problems in the various stadiums from a practical and organizational and also emotional point of view. Caring for dementia sufferers is a highly demanding task both emotionally and physically [7]. All of these aspects can activate new conflicts with tiredness, with economic problems or with decisions to take. They can also reactivate ancient tensions that sometimes lead to definitive breakups [8] [9] [10].

Behavioral and psychological symptoms of dementia (BPSD) affect the majority of patients at the same point in the progression of the disease [11] [12] [13] [14], involving the patient, the family caregiver and their environment.

BPSD are more stressful to caregivers than cognitive or functional decline, because they are felt as the most difficult to manage and have a negative impact on the relationships between the caregiver, patient and family [1] [14].

Moreover caregivers differ in their emotional responses to BPSD. The caregiver's perception of the patient's problems is more important than the problem linked to the patient's behavior "per se" [15] [16]. The caregiver's interpretation reaction may be adequate or inadequate. So the subjective factors and individual differences among caregivers in the caring experience and in coping are fundamental [17].

The personality characteristics affect the processes that individuals use to appraise stressful events and predispose them to cope in certain ways when they confront these events [7] [17] [18].

Personality also has significant direct and indirect effects on mental health and direct effects on physical health. 
The objective and subjective burden of care and psychiatric morbidity [7] [17], such as depression, is associated with specific psychological characteristics of the caregiver [19] [20] [21], and influence some factors of the caregivers themselves, such as their coping strategies to deal with the symptoms and meaning of the illness [19] [20] [21].

Personality traits are an indicator of global individual propensity to experience negative influences. Individuals who show intrapsychic problematic dimensions tend to show behaviors such as "emotional imbalance, unrealistic ideas, desires and needs excessive or inappropriate coping responses", while those who are "calm, relaxed, resilient, secure, non-emotive, self-satisfied" [20] [21] [22] [23] may present better coping. Moreover caregiving is at high risk for social isolation [24] [25].

The tendency to isolation and low optimism are factors that facilitate depression [26]. So the individual propensity to social interaction and activities is another important aspect. Caregiver's extraversion decreases both burden and depression. On the basis of all these considerations it could be useful that caregivers participate in groups of psychological support [27] [28] [29] [30] [31] that have the purpose of furnishing coping tools to decrease the levels of stress and to offer a support finalized to the acceptance of the illness, to the elaboration of the change of emotional reactions such as anxiety, sense of guilt, depression, anger, embarrassment and loneliness. Everything contributes to improving the quality of life of the whole family nucleus, to favour the permanence of the patient in his/her own domicile and to raise the awareness of the families of not being abandoned in this painful situation. The inclusion of caregivers' personality traits studies would increase the knowledge of their role and hopefully contribute to improve the quality of life of both caregiver and patient. The specific aim of our study was to establish whether a difference exists in the intrapsychic and interpersonal dynamics and in the prevalence of depression of caregiving and not caregiving subjects. The use of the SASB model in the present study contributes to the knowledge of intrapsychic and interpersonal processes in order to plan a more suitable psychotherapeutic intervention for the caregiver as part of multidisciplinary care.

\section{Methods}

Sampling-Choice of Caregivers (CG): The study groups were composed of $n=$ 75 caregivers (male and female-age range: 45 - 75 years, caregiving from 2 and half to 5 years, sampled in a casual way (from a total of 195 CG), relatives (wife, husband, son, daughter and so on) of patients affected by Alzheimer disease. Control group n. 104 healthy subjects are not caregivers (nCG). These subjects were sampled respecting the following conditions: they were healthy and did not provide sick family assistance at least for the last 5 years.

The caregivers were selected from the Alzheimer Disease Daily Center of Florence, and the Department of Neurology, INRCA-IRCCS Ancona Italy (2017, 
2018). The research project was accepted by the Ethical Committee.

All the subjects studied (CG and nCG) were subdivided on the basis of the following independent variables: sex, age marriage status and educational level and were homogeneous for these variables: no significant differences emerged between the two samples (Table 1).

All the patients were affected by Dementia of Alzheimer with clinical diagnosis through Tac. The diagnosis of Alzheimer Disease was effected respecting the criteria of the DSM-IV while its degenerative nature was identified following the standardized criterions NINCDS-ADRDA for the diagnosis of illness of Alzheimer [32]. In particular all criteria were satisfied for the diagnosis of "probable Illness of Alzheimer" (with the exception of the liquorale examination). All the patients in this study showed level 6 of the Functional 6 Assessment Staying Test.

Inclusion criteria included an age greater than 45 years (adult 45 - 59 yrs) and also elderly CG (60 - 75 yrs). Exclusion criteria included: refusal to participate; inability to provide informed consent; previous history of depression; use of psychotropic drugs (all, including antidepressant).

One hundred and twenty three caregivers were approached in the clinic by the physician and asked to participate in the study. All participants signed a consent form regarding study protocol after detailed explanation by the physician at the day center for Alzheimer disease patients.

Only ninety caregivers decided to participate and to fill out and sign the consent form. The caregivers were free to complete the questionnaire either in the center or at home.

Subjects who decided to complete forms at home were given a self-addressed,

Table 1. Demografic charachteristic of caregivers and not-caregivers.

\begin{tabular}{ccc}
\hline & Caregivers characteristics & Not caregivers characteristics \\
\hline Sex & n. 73 & n. 104 \\
Female 68\% & Male 32\% & Female 69\% \\
Mean (SD) Age, Years & 63 & 61 \\
Marital Status & & \\
Single & $9.30 \%$ & $10 \%$ \\
Married & $79.80 \%$ & $77 \%$ \\
Widow & $8.50 \%$ & $9.80 \%$ \\
Divorced & $2.30 \%$ & $3.20 \%$ \\
Educational Level & & $40.30 \%$ \\
Elementary School & $39.50 \%$ & $24.20 \%$ \\
Middle School & $22.90 \%$ & $27 \%$ \\
High School & $27.05 \%$ & $8.50 \%$ \\
University & $9.30 \%$ &
\end{tabular}


stamped envelope to return the forms. Fifteen patients didn't answer all the questions in the questionnaires: it was therefore decided not to consider them for the analysis. All subjects (case and control groups), were asked to complete the following psychological and psychosocial questionnaires:

1) Social schedule, including data on gender, age, marital status, educational level, profession.

2) Structural Analysis of Social Behavior (SASB) Model-Anint A Questionnaire (intrapsychic factors) by L.S. Benjamin [33] [34] (Appendix). The Italian version ASCI (Structural Analysis of Interpersonal Behavior) by P. Scilligo, is validated on the basis of DSMIV and on the Italian population.

This test evaluates the mental processes of the personality structure at an intrapsychic and interpersonal level. It includes 36 descriptive items of two series of eight clusters, respectively, of intrapsychic (Oneself) and interpersonal (Other) experiences. The test assesses intrapsychic and interpersonal components of the personality andpredicts the evolution of the mental structure following interpersonal interactions.

Interviewed subjects had to respond to 36 items in the questionnaire describing their intrapsychic behaviours during the last year (e.g., "I neglect myself, don't try to develop good skills, ways of being"; "I practice and work on developing worthwhile skills, ways of being"; "I think up ways to hurt and destroy myself. I am my own worst enemy"). They are rated on a 10-point scale ranging from 0 (Never) to 10 (All the time). The SASB-Form-A questionnaire describes the structure of personality from normal to pathological. The 36 questions of Form-A are grouped by a specific score correction in 8 clusters $(\mathrm{Cl})$ of intrapsychic "Oneself” and interpersonal "Other" experience. The 8 clusters of "Onself" and "Other" are complementary and opposed (respectively $\mathrm{Cl} 1$ and $\mathrm{Cl} 5 ; \mathrm{Cl} 2$ and $\mathrm{Cl}$; $\mathrm{Cl} 3$ and $\mathrm{Cl} 7 \mathrm{Cl} 4$ and $\mathrm{Cl}$ ): high levels in Clusters 1, 2, 3, 4 corresponds to low levels respectively in Clusters 5, 6, 7, 8) (Appendix).

Description of the 8 clusters of "Oneself"-Intrapsychic and interpersonal experience (Appendix):

SASB Cluster $(\mathrm{Cl}) 1=$ Autonomy-Assertive and Separating.

SASB Cluster $(\mathrm{Cl}) 2=$ Autonomy and Love-Self-Accepting and Exploring.

SASB Cluster $(\mathrm{Cl}) 3=$ Love Self-Supporting and Appreciative.

SASB Cluster $(\mathrm{Cl}) 4=$ Love and Control-Self-Care and Development.

SASB Cluster $(\mathrm{Cl}) 5=$ Control Self-Regulating and Controlling.

SASB Cluster $(\mathrm{Cl}) 6=$ Control and Hate Self-Critical and Oppressive.

SASB Cluster $(\mathrm{Cl}) 7=$ Hate Self-Refusing and Annulling.

SASB Cluster $(\mathrm{Cl}) 8$ = Hate and Autonomy-Self-Negligent and Mentally Absent.

3) CDQ tests by Cattell [35] have been used as methods of Self-report which describe depression, respectively. The range is subdivided as follows:

0 - 3 indicates absence of anxiety or depression; 4 - 7 indicates medium to medium-high level of anxiety and depression, and 8 - 10 indicates a high level of 
depression.

\section{Statistical Analysis}

The data were analyzed using SPSS 11.5 (SPSS Inc. Chicago, Illinois). Variance analysis (ANOVA) was applied to evaluate the differences between the two groups of subjects (case and control groups) on the Scales SASB and CDQ.

The maximum significant level considered in this study was 0.05 . To achieve power of 0.80 and a medium effect size, a sample of 60 was required to detect a significant model.

\section{Results}

\subsection{SASB Questionnaire (Intrapsychic Behaviours) and CDQ}

The results show a significant difference for the varying depression between the study group of caregivers and the control group of healthy people not assistant of family patients $(\mathrm{p}<0.001)$.

In terms of intrapsychic modalities the two groups (caregivers (CG) and control group (nCG) obtained different scores in all clusters except SASB Cl 8 Hate and autonomy-self-negligent and mentally absent. Patients are not likely to neglect themselves and their needs.

Significant differences were found in the intrapsychic processes of SASB questionnaire (Figure 1) of the two groups CG and nCG in the following clusters: $\mathrm{Cl} 1=$ Autonomy - Assertive and Separating $(\mathrm{F}=26.209 ; \mathrm{p}<0.001) ; \mathrm{SASB} \mathrm{Cl} 2=$ Autonomy and Love $(\mathrm{F}=15,528 ; \mathrm{p}<0.001)$, SASB CL3 $=$ Love $(\mathrm{F}=51,173, \mathrm{p}<$ $0.001)$; SASB Cl $4=$ Love and Control $(\mathrm{F}=35.647 ; \mathrm{p}<0.001)$, SASB CL5 $=$ Control $(\mathrm{F}=6005 ; \mathrm{p}=0.015)$, SASB Cl6 $=$ Control and Hate $(\mathrm{F}=36,935 ; \mathrm{p}<0.001)$, SASB Cl7 $=$ Hate $(\mathrm{F}=21,577 ; \mathrm{p}<0.001)$.

SASB Cl1 = Autonomy-Assertive and Separating. The caregiver may be not spontaneous with self-acceptance and pleasure in his/her experience or could be disoriented and give little weight to problems and important choices in life.

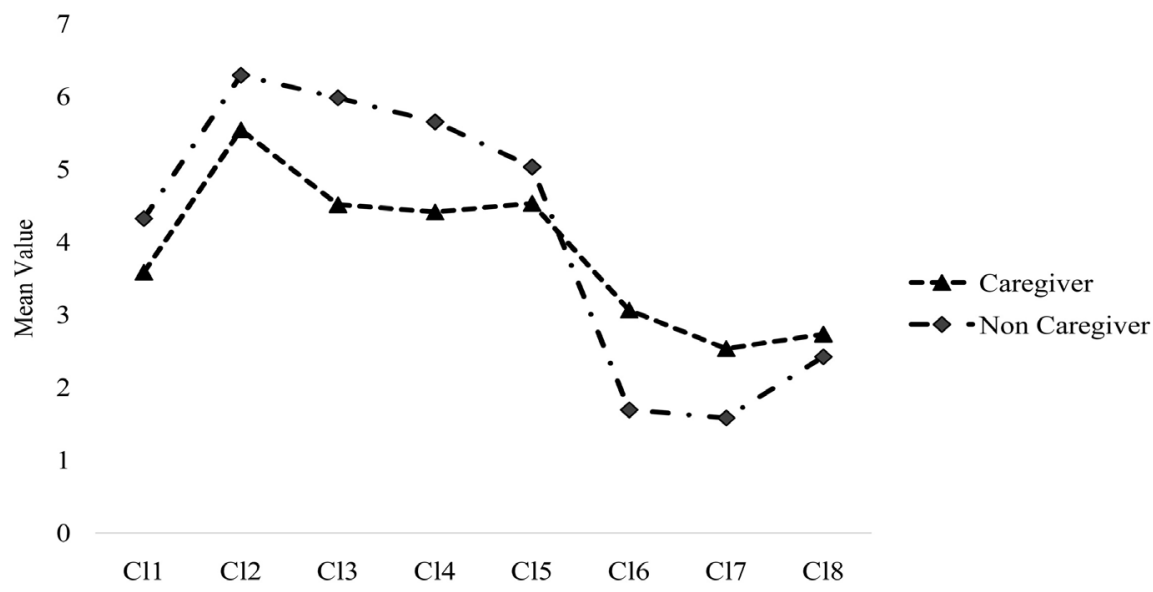

Figure 1. Differences between SASB clusters mean values of caregiver and non caregiver groups. 
SASB Cl2 = Self Acceptance: Caregivers manifested lower levels of self-confidence and of acceptance of their own emotions than control group;

SASB Cl3 = Self Supporting and Appreciate (Self-Esteem): Caregivers have a worse attitude toward themselves and toward self-care than control group.

They were less likely to show self-esteem and to care for themselves in the presence of stressful situations.

SASB Cl4 = Love and Control-Self-Care and Development.

Caregivers have a worse attitude in being positively self-constructive, and using energy to obtain what is needed and desired than control group.

SASB Cl5 = Self-Control: Caregivers scores show more self-control than healthy not caregiving subjects. In particular, these subjects are more prone to manage and program themselves towards pre-fixed aims. In other words they express less spontaneity and flexibility.

SASB Cl6 = Self-Criticism and Oppression: Caregivers are more likely to self-abuse and self-criticism than control group.

SASB Cl7 = Hate and Self Destruction: Caregivers are more likely to engage in self-destructive behaviors in the physical and emotional dimensions.

Synthesizing caregivers are less prone to be in touch with their own feelings and emotions. They are not satisfied with themselves, their lives and their entourages and show difficulties to cope with stress. They don't always manifest self-appreciation and self-esteem and may not be able to achieve emotional and psychic equilibrium in the presence of stress. In addition they are less likely to protect themselves and to utilize crisis and stress for their own emotional development.

In presence of stress they may likely become disoriented and engage in behaviors that may be self-defeating and self-abusive. They show serious difficulties in facing and accepting their own emotions. The presence of self-criticism creates an additional problem.

Because of this poor coping they may be more subject to depression and depressive moods.

The profile of the SASB model shows high likelihood of depression in caregivers group.

Moreover these subjects display low assertiveness, and low ability to accept themselves and support themselves (to treat, care for, console and consolidate). They may be oppressive towards themselves and may accuse themselves of inadequacy, evoking feelings of guilt and shame, which purport low self-esteem. In general, caregivers exercise greater self-control aimed to specific objectives, exhausting themselves toward predetermined goals compared to control group.

The results in Anint A questionnaire of SASB Model show that caregivers present a minor state of depression without reaching a level of major depression.

\subsection{Interpersonal Behaviors}

On the basis of these intrapsychic processes it is possible to describe the differ- 
ences in interpersonal behaviors between caregivers and control group. The significant differences are reached in the following clusters: SASB Cl1-Autonomy-Liberating and Forgetting $(\mathrm{F}=26,209 ; \mathrm{p}<0.001)$; SASB Cl2-Autonomy and Love-Confirming and Understanding $(\mathrm{F}=15,528$; $\mathrm{p}<0.001)$; SASB $\mathrm{Cl} 3$-Love-Caring and Consoling ( $\mathrm{F}=51,173 ; \mathrm{p}<0.001)$; SASB Cl4-Love and Control-Helping and Protecting $(\mathrm{F}=35,647 ; \mathrm{p}<0.000)$; SASB Cl5-Control-Looking after and Managing $(\mathrm{F}=6005 ; \mathrm{p}=0.015)$; SASB Cl6-Control and Hate-Belittling and Blaming $(F=36,935 ; \mathrm{p}<0.001)$; SASB $\mathrm{Cl} 7-$ Hate-Assaulting and Refusing $(\mathrm{F}=21,577 ; \mathrm{p}<0.001)$. In $\mathrm{Cl} 8$ a significant difference is not reached.

Caregivers' Interpersonal Profile: These patients don't fully promote independence in the relationship with others, by expressing trust and encouraging other people's independent identity; they are not always appreciative and empathic toward accepting other people's difference of opinion; they do not always search to be close to others and do not reach a real intimacy; they control others, by reminding them what they should think, do and say. In extreme cases they may seriously threaten others.

They tend to control other people in a positive and negative way and may express behaviors of belittling, blaming or manipulating others. In extreme cases they may ignore and neglect the needs and interests of others.

Correlations-SASB and Depression-Caregiver: The results show significant correlations between depression (CDQ) and SASB Clusters.

CDQ (medium high levels): SASB Cl1 $(\mathrm{r}=-0.472 ; \mathrm{p}<0.001)$; SASB Cl2 $(\mathrm{r}=$ $-0.354 ; \mathrm{p}=0.016)$; SASB Cl3 ( $\mathrm{r}=-0.469 ; \mathrm{p}<0.001)$; SASB CL6 $(\mathrm{r}=0.6518 ; \mathrm{p}<$ 0.001); CL7 ( $\mathrm{r}=0.409 ; \mathrm{p}<0.005)$; CL8 ( $\mathrm{r}=0.300 ; \mathrm{p}<0.043)$.

More depression is correlated with less autonomy and love, and with more control. The caregivers' behaviors of not being spontaneous with self-acceptance and pleasure in their experience or being disoriented give little weight to problems and important choices in life is correlated with depression. The caregivers' behaviors of self-criticism and oppression and tendency to self-neglecting behaviours (of needs at emotional and physical levels) are correlated with medium high levels of depression. Caregivers are less likely to show self-care and self-esteem in the presence of stressful situations. These behaviors are correlated with depression (medium high levels).

\section{Discussion}

Compared to nCR, caregivers presented a higher level of depression (medium-high). In addition, there was a substantial difference in the number of intrapsychic and interpersonal attitudes. CG presented less autonomy in their choices and lower acceptance of their own feelings. These individuals were less spontaneous in their behavior and showed difficulties ingetting in touch with and accepting their deeper feelings. Being unappreciative of themselves, they showed low capacity to treat, console, care for and forgive themselves. If left un- 
treated, these attitudes could hamper the patient's ability to reach and maintain a good quality of life.

They could be unabletocope with the stress that the condition of assistance to their sick family memberinvolves.

So our results are in agreement with the statement that assisting a person with dementia can bring emotional resources to the limit and lead to anxious-depressive moods (Schulz, 2008; Papastavrou, 2012). Moreover these intrapsychic problems and depression shows that the caregiver may no longer be able to assist the patient.

These considerations suggest that the screening of intrapsychic factors and depression levels may be an indicator of needs of support (psychotherapeutic intervention, social services support).

Based on the intrapsychic profile (SASB) which emerged, specific psychotherapeutic intervention could be necessary (Benjamin, 2006) for facilitating contact, self-awareness elaboration and integration of emotional experiences (passive adaptation, low self-affirmation, self-criticism), in order to change the life style and to encourage resources necessary for a successful adaptation to the family patient disease condition. So we hypothesize that the changes of intrapsychic behaviors could contribute in decreasing depression and stress linked to the burden.

Sörensen affirms: "Caregiver psychological interventions are effective, but some interventions have primarily domain-specific effects rather than global effects. The differences between intervention types and moderators suggest ways of optimizing interventions".

The use of meditation-based therapies, including Mindfulness Therapy MBSR, the positive effects of which are now recognized by the scientific literature, should be integrated with the reprocessing of the experience of the intra-psychic conflicts that underlie maladaptive lifestyle-related disease.

We think that in this context the use of Holistic Psychotherapy and Mindfulness it could be the most appropriate intervention given the emerged intrapsychic problems.

So it is necessary to make the following considerations: the psychotherapeutic intervention to be effective should be an integrated approach (Behavioral Therapy), Holistic Psychotherapy (Therapeutic Psychosyntesis), meditation practices (Mindfulness-Transpersonal Psychology), relaxation techniques and guided imagery (Brief Psychotherapy) and so on (Sörensen, 2002).

The psychotherapeutic intervention must take account of intra-psychic problems of caregivers of patients affected by Alzheimer's Disease. Therefore a screening of intrapsychic problems is desirable in clinical practice. Given the described personality problems, it can be concluded that a psychotherapeutic approach ( psychotherapy in group or individual session) could, in order to be effective over time and to prevent stress, the sense of uneasiness and the state of depression (Roth, 2005; de Rotrou, 2011; Ducharme, 2011; Negovanska, 2011) address the following issues: 
1) to give the possibility to elaborate emotional reactions and integrate them; to prevent or face the levels of stress and to realize an acceptance of the illness (Benjamin).

2) to teach the abilities of elaboration of the change of roles and the emotional reactions towards the patient's personality degeneration (anxiety, sense of guilt, anger, embarrassment, sense of loneliness), and of decoding and understanding the patient's antisocial behaviors (García-Alberca, 2012; de Rotrou, 2011; Ducharme, 2011; Hooker, 2002).

3 ) to integrate the emotional uneasiness for the restoration of a good quality of life within the family nucleus (Benjamin, 2006).

The consequence of this intervention could be the permanence of the patient in the family nucleus. Stress management and reduction is important for the caregiver in order to maintain or restore mental and physical health.

The other important aspect is to create services of practical support that raise the relief load and are of support in this sense. It is very important, especially in Italy, where it is mostly the family that takes care of the patient affected by Alzheimer's disease. The caregiver must not feel alone in this situation (Nápoles, 2010; de Rotrou, 2011).

In conclusion the main finding of the present study is that several dimensions of the caregiver's personality are very problematic and may strongly influence the burden and depressive symptoms of the caregiver.

Our study has several limitations. The first limitation is the small sample of caregivers.

This does not allow us to compare adults and elderly caregivers. Moreover our results provide a snapshot of distress and intrapsychic mechanisms during the caregiving phases without distinguishing time of assistance. The results may differ during the caregiving phases or at other points in the disease journey. Because of the small sample we did not have the data to compare the exact length of time which elapsed from the beginning of assistance.

Another sampling bias was present in the data because all the subjects attended only two institutions and thus were not representative of caregivers in general.

So further studies are necessary especially in the experimentation of psychotherapeutic interventions.

\section{Conflicts of Interest}

The authors declare no conflicts of interest regarding the publication of this paper.

\section{References}

[1] Berger, G., Bernhardt, T., Weimer, E., et al. (2005) Longitudinal Study on the Relationship between Symptomatology of Dementia and Levels of Subjective Burden and Depression among Family Caregivers in Memory Clinic Patients. Journal of Geriatric Psychiatry and Neurology, 18, 119-128. 
https://doi.org/10.1177/0891988704273375

[2] Papastavrou, E., Kalokerinou, A., Papacostas, S.S., et al. (2007) Caring for a Relative with Dementia: Family Caregiver Burden. JAN, 58, 446-457.

https://doi.org/10.1111/j.1365-2648.2007.04250.x

[3] Mohamed, S., Rosenheck, R., Lyketsos, C.G., et al. (2010) Caregiver Burden in Alzheimer Disease: Cross-Sectional and Longitudinal Patient Correlates. The American Journal of Geriatric Psychiatry, 18, 917-927. https://doi.org/10.1097/JGP.0b013e3181d5745d

[4] Schulz, R., McGinnis, K.A., Zhang, S., et al. (2008) Dementia Patient Suffering and Caregiver Depression. Alzheimer Disease \& Associated Disorders, 22, 170-176. https://doi.org/10.1097/WAD.0b013e31816653cc

[5] Mausbach, B.T., Chattillion, E., Roepke S.K., et al. (2012) A Longitudinal Analysis of the Relations among Stress, Depressive Symptoms, Leisure Satisfaction, and Endothelial Function in Caregivers. Health Psychology, 31, 433-440. https://doi.org/10.1037/a0027783

[6] García-Alberca, J.M., Cruz, B., Lara, J.P., et al. (2013) The Experience of Caregiving: The Influence of Coping Strategies on Behavioral and Psychological Symptoms in Patients with Alzheimer's Disease. Aging \& Mental Health, 17, 615-622. https://doi.org/10.1080/13607863.2013.765833

[7] Rocca, P., Leotta, D, Liffredo, C., et al. (2010) Neuropsychiatric Symptoms Underlying Caregiver Stress and Insight in Alzheimer's Disease. Dementia and Geriatric Cognitive Disorders, 30, 57-63. https://doi.org/10.1159/000315513

[8] García-Alberca, J.M., Cruz, B., Lara, J.P., et al. (2012) Disengagement Coping Partially Mediates the Relationship between Caregiver Burden and Anxiety and Depression in Caregivers of People with Alzheimer's Disease. Results from the MÁLAGA-AD Study. Journal of Affective Disorders, 136, 848-856. https://doi.org/10.1016/j.jad.2011.09.026

[9] Papastavrou, E., Charalambous, A., Tsangari, H., et al. (2012)The Burdensome and Depressive Experience of Caring: What Cancer, Schizophrenia, and Alzheimer's Disease Caregivers Have in Common. Cancer Nursing, 35, 187-194. https://doi.org/10.1097/NCC.0b013e31822cb4a0

[10] Epstein-Lubow, G., Gaudiano, B., Darling, E., et al. (2012) Differences in Depression Severity in Family Caregivers of Hospitalized Individuals with Dementia and Family Caregivers of Outpatients with Dementia. The American Journal of Geriatric Psychiatry, 20, 815-819. https://doi.org/10.1097/JGP.0b013e318235b62f

[11] Engelborghs, S., Maertens, K., Nagels, G., et al. (2005) Neuropsychiatric Symptoms of Dementia: Cross-Sectional Analysis from a Prospective, Longitudinal Belgian Study. International Journal of Geriatric Psychiatry, 20, 1028-1037. https://doi.org/10.1002/gps.1395

[12] Campbell, P., Wright, J., Oyebode, J., et al. (2008) Determinants of Burden in Those Who Care for Someone with Dementia. International Journal of Geriatric Psychiatry, 23, 1078-1085. https://doi.org/10.1002/gps.2071

[13] Lyketsos, C.G., Lopez, O., Jones, B., et al. (2002) Prevalence of Neuropsychiatric Symptoms in Dementia and Cognitive Impairment: Results from the Cardiovascular Health Study. JAMA, 25, 1475-1483. https://doi.org/10.1001/jama.288.12.1475

[14] Vugt, M.E., Stevens, F., Aalten, P., et al. (2003) Behavioural Disturbances in Dementia and Quality of the Marital Relationship. International Journal of Geriatric Psychiatry, 18, 149-154. https://doi.org/10.1002/gps.807

[15] Mausbach, B.T., Roepke, S.K., Chattillion, E.A., et al. (2012) Multiple Mediators of 
the Relations between Caregiving Stress and Depressive Symptoms. Aging \& Mental Health, 16, 27-38. https://doi.org/10.1080/13607863.2011.615738

[16] Boutoleau-Bretonnière, C. and Vercelletto, M. (2009) Caregiver Burden in Dementia: Relationships with the Activities of Daily Living, Behavioral, and Psychological Symptoms. Psychologie \& NeuroPsychiatrie du Vieillissement, 7, 15-20.

[17] Hooker, K., Frazier, L.D. and Monahan, D.J. (1994) Personality and Coping among Caregivers of Spouses with Dementia. Gerontologist, 34, 386-392. https://doi.org/10.1093/geront/34.3.386

[18] Hooker, K., Monahan, D.J., Bowman, S.R., et al. (1998) Personality Counts a Lot: Predictors of Mental and Physical Health of Spouses Caregivers in Two Disease Groups. The Journals of Gerontology. Series B, Psychological Sciences and Social Sciences, 2, 73-85. https://doi.org/10.1093/geronb/53B.2.P73

[19] Jang, Y., Clay, O.J. and Roth, D.L. (2004) Neuroticism and Longitudinal Change in Caregiver Depression: Impact of a Spouse-Caregiver Intervention Program. Gerontologist, 44, 311-317. https://doi.org/10.1093/geront/44.3.311

[20] Shurgot, G.R. and Knight, B.G. (2005) Influence of Neuroticism, Ethnicity, Familism, and Social Support on Perceived Burden in Dementia Caregivers: Pilot Test of Transactional Stress and Social Support Model. The Journals of Gerontology. Series B, Psychological Sciences and Social Sciences, 60, 331-334.

https://doi.org/10.1093/geronb/60.6.P331

[21] Sink, K.M., Covinsky, K.E., Barnes, D.E., et al. (2006) Caregiver Characteristics Are Associated with Neuropsychiatric Symptoms of Dementia. Journal of the American Geriatrics Society, 54, 796-803. https://doi.org/10.1111/j.1532-5415.2006.00697.x

[22] García-Alberca, J.M., Cruz, B., Lara, J.P., et al. (2012) Anxiety and Depression Are Associated with Coping Strategies in Caregivers of Alzheimer's Disease Patients: Results from the MALAGA-AD Study. International Psychogeriatrics, 24, 1325-1334. https://doi.org/10.1017/S1041610211002948

[23] Watson, L.C., Lewis, C.L., Moore, C.G., et al. (2011) Perceptions of Depression among Dementia Caregivers: Findings from the CATIE-AD Trial. International Journal of Geriatric Psychiatry, 26, 397-402. https://doi.org/10.1002/gps.2539

[24] Sherwood, P., Given, C., Given, B. and Von Eye, A. (2005) Caregiver Burden and Depressive Symptoms: Analyses of Common Outcomes in Caregivers of Elderly Patients. Journal of Aging and Health, 17, 125-147. https://doi.org/10.1177/0898264304274179

[25] Roth, D.L., Mittelman, M.S., Clay, O.J., et al. (2005) Changes in Social Supports as Mediators of the Impact of a Psychosocial Intervention for Spouse Caregivers of Persons with Alzheimer's Disease. Psychology and Aging, 20, 634-644.

https://doi.org/10.1037/0882-7974.20.4.634

[26] Thomas, P., Lalloué, F., Preux, P.M., et al. (2006) Dementia Patients Caregivers Quality of Life: The PIXEL Study. International Journal of Geriatric Psychiatry, 21, 50-56. https://doi.org/10.1002/gps.1422

[27] De Rotrou, J., Cantegreil, I., Faucounau, V., et al. (2011) Do Patients Diagnosed with Alzheimer's Disease Benefit from a Psycho-Educational Programme for Family Caregivers? A Randomised Controlled Study. International Journal of Geriatric Psychiatry, 26, 833-842. https://doi.org/10.1002/gps.2611

[28] Ducharme, F.C., Lévesque, L.L., Lachance, L.M., et al. (2011) Learning to Become a Family Caregiver Efficacy of an Intervention Program for Caregivers Following Diagnosis of Dementia in a Relative. Gerontologist, 51, 484-494.

https://doi.org/10.1093/geront/gnr014 
[29] Nápoles, A.M., Chadiha, L., Eversley, R., et al. (2010) Developing Culturally Sensitive Dementia Caregiver Interventions: Are We There Yet? American Journal of Alzheimer's Disease \& Other Dementias, 25, 389-406. https://doi.org/10.1177/1533317510370957

[30] Sörensen, S., Pinquart, M. and Duberstein, P. (2002) How Effective Are Interventions with Caregivers? An Updated Meta-Analysis. Gerontologist, 42, 356-372. https://doi.org/10.1093/geront/42.3.356

[31] Negovanska, V., Hergueta, T., Guichart-Gomez, E., et al. (2011) Beneficial Effect of a Cognitive Behavioral and Multidisciplinary Program in Alzheimer Disease on Spouse Caregiver Anxiety. Revista de Neurología, 167, 114-122. https://doi.org/10.1016/j.neurol.2010.07.002

[32] Varma, A.R., Snowden, J.S., Lloyd, J.J., et al. (1999) Evaluation of the Nincds-Adrda Criteria in the Differentiation of Alzheimer's Disease and Frontotemporal Dementia. Journal of Neurology, Neurosurgery, and Psychiatry, 66, 184-188. https://doi.org/10.1136/jnnp.66.2.184

[33] Benjamin, L.S., Rothweiler, J.C. and Critchfield, K.L. (2006) The Use of Structural Analysis of Social Behavior (SASB) as an Assessment Tool. Annual Review of Clinical Psychology, 2, 83-109. https://doi.org/10.1146/annurev.clinpsy.2.022305.095337

[34] Critchfield, K.L. and Benjamin, L.S. (2010) Assessment of Repeated Relational Patterns for Individual Cases Using the SASB-Based Intrex Questionnaire. Journal of Personality Assessment, 92, 480-489. https://doi.org/10.1080/00223891.2010.513286

[35] Krug, S.E. and Laughlin, J.E. (1978) Questionario di autovalutazione CDQ (IPAT Depression Scale): Manuale. Adattamento italiano a cura di Marcello Novaga e Arrigo Pedon (ed.). Organizzazioni speciali, Firenze. 


\section{Appendix}

\section{SASB Model}

SASB-Anint A questionnaire evaluates the mental processes of the personality structure at an intra-psychic and interpersonal level. It includes 36 descriptive items of two series of 8 clusters, respectively of intra-psychic (Oneself) and interpersonal (Other) experiences. The test assesses intra-psychic and interpersonal components of the personality and predicts the evolution of the mental structure following interpersonal interactions.

The 36 questions provide an exhaustive picture of intra-psychic experience from which the interpersonal one can be inferred.

The 8 clusters of "Onself" and "Other" are both complementary.

SASB-Cluster $(\mathrm{Cl}) 1=$ Autonomy-Assertive and Separating.

SASB-Cluster $(\mathrm{Cl}) 2=$ Autonomy and Love-Self-Accepting and Exploring.

SASB-Cluster $(\mathrm{Cl}) 3$ = Love-Self-Supporting and Appreciative.

SASB-Cluster $(\mathrm{Cl}) 4=$ Love and Control-Self-Care and Development.

SASB-Cluster $(\mathrm{Cl}) 5=$ Control-Self-Regulating and Controlling.

SASB-Cluster $(\mathrm{Cl}) 6=$ Control and Hate-Self-Critical and Oppressive.

SASB-Cluster $(\mathrm{Cl}) 7=$ Hate-Self-Refusing and Annulling.

SASB-Cluster $(\mathrm{Cl}) 8=$ Hate and Autonomy-Self-Negligent and Mentally Absent.

The variables of the two dimensions: "Oneself" and "Other" are complementary: a modality of interpersonal experience corresponds to an intra-psychic experience.

SASB Model-Rules for determining attention:

1) Intrapsychic: ("Oneself"-inward attention): attention is turned inwards. Transitive action with the person himself is as the subject and at the same time as the object. This form can be either active or passive. The person does things for him/herself-or thinks of something concerning him/herself.

\section{Intrapsychic Behaviors-8 Clusters}

a) SASB-Cl $1=$ Autonomy-Assertive and Separating. This type does what seems right on the basis of what he considers is necessary at the time. The attitude may be spontaneous, with self-acceptance, and pleasure in the experience. Or it could be disoriented and the person gives little weight to problems and important choices in life.

b) SASB-Cl $2=$ Autonomy and Love-Self-Accepting and Exploring. This type accepts and reacts to his deepest feelings, feeling solid, integrated and "together". The desire to be open to feelings generally indicates a state of self-satisfaction and acceptance of weak and strong points.

c) SASB-Cl 3 = Love-Self-Supporting and Appreciative. This type is deeply appreciative of himself and is able to treat, care for, console and reconsolidate himself. He has a capacity for self-esteem and in extreme cases for self-adoration.

d) SASB-Cl $4=$ Love and Control-Self-Care and Development. This type 
protects and realistically examines himself and has the capacity to be positively self-constructive, actively developing his abilities and other important qualities for self-growth. This can imply using much energy to obtain what is needed and desired.

e) SASB Cl5= Control-Self-Regulating and Controlling. This type can control himself. Great self-control is exercised for chosen objectives. This may include paying attention to behavior in order to ensure conforming to ideals, including great activity programmed to reach objectives.

f) SASB Cl6= Control and Hate-Self-Critical and Oppressive. This type oppresses himself and may accuse himself of inadequacy, evoking feelings of self-guilt and shame. Feelings of uncertainty and guilt can be used for false induction to what is recognized as not being useful to the person. This could be self-punitive behaviour, sometimes destructive enough as to call for therapeutic intervention.

g) SASB Cl7= Hate-Self-Refusing and Annulling. This self-destructive type may ignore illness and wounds, exhaust himself and become completely listless. This implies self-refusal and self-deprivation and generally self-inflicted cruelty. Such self-destructive behaviour calls for serious qualified psychotherapeutic intervention.

h) SASB-Cl $8=$ Hate and Autonomy-Self-Negligent and Mentally Absent. This type may daydream, subsequently not developing abilities and potentials to the full. In extreme cases he may have unreasonable and unjustified ideas about himself and behave without any criterion, falling into self-destructive situations. In these cases it could be beneficial to examine the danger of self-destructive behavior with a therapist.

i) Interpersonal ("Other"-attention towards the other): attention is turned outwards, doing something for, or with reference, to others. This does not include what others do for the person, but implies an action involving the other as the direct object: an action is expressed influencing someone or something and a direct object is needed to complete the sequence. Actions start with a proposer and have an effect on someone or something.

Attention towards the other can be either active or passive: the phrase "you hit me" and "I was hit by you" are equivalent ASCI evaluations. In both cases the action is expressed by the one same person and results in affecting the other.

\section{Interpersonal Behaviors-8 Clusters}

a) Autonomy-Liberating and Forgetting. This type emancipates, liberates the other by expressing trust and encouraging his independent identity. However this emancipation may include elements of neglect and forgetfulness.

b) Autonomy and Love-Confirming and Understanding. This type is appreciative, understanding and confirming, being empathic toward the other. Behaviour patterns which may also be present include treating the other justly, listening to him attentively even if there are differences of opinion. 
c) Love-Caring and Consoling. This type is described as caring, attentive, consoling and desiring to be close to the other person. Among lovers this behaviour can also lead to sexual tenderness.

d) Love and Control-Helping and Protecting. This type is described as someone who actively helps the other by protecting, sustaining, advising and even teaching him. This kind of help could, if extreme, turn to spoiling and indulging behaviour.

e) Control-Looking after and Managing. This type controls the other, reminding him what should be thought, done and said, for the "good of the other person". Other less positive forms of control can be expressed as limiting freedom and forcing the other to conform to specific rules or orders.

f) Control and Hate-Belittling and Blaming. This type is described as belittling, blaming or manipulating the other in a deceitful way. He may try to make the other admit to faults and in extreme cases demand revenge and threaten with punishment.

g) Hate-Assaulting and Refusing. This type can seriously threaten or hurt the other. Extreme behaviour may include physical or moral assault with destructive intention. Other forms might be: stinginess with necessities, ignoring, refusal with anger or taking advantage of the other person.

h) Hate and Autonomy-Ignoring and Forgetting. This type is described as basically ignoring and neglecting the needs and interests of the other. This implies disattention and can produce or derive from unusual concepts and perceptions. Such treatment of the other can appear illogical and senseless. This type could abandon the other in more critical moments. 